\title{
Some advances in the theory of voting systems based on experimental algorithms ${ }^{\star}$
}

\author{
Josep Freixas $^{1}$ and Xavier Molinero ${ }^{2}$ \\ Escola Politècnica Superior d'Enginyeria de Manresa E-08242 Manresa, Spain. \\ Universitat Politècnica de Catalunya. \\ ${ }^{1}$ Dept. de Matemàtica Aplicada 3. E-mail: josep.freixas@upc.edu. \\ ${ }^{2}$ Dept. de Llenguatges i Sistemes Informàtics. E-mail: molinero@lsi.upc.edu.
}

\begin{abstract}
In voting systems, game theory, switching functions, threshold logic, hypergraphs or coherent structures there is an important problem that consists in determining the weightedness of a voting system by means of trades among voters in sets of coalitions. The fundamental theorem by Taylor and Zwicker [8] establishes the equivalence between weighted voting games and $k$-trade robust games for each positive integer $k$. Moreover, they also construct, in [9], a succession of games $G_{k}$ based on magic squares which are $(k-1)$-trade robust but not $k$-trade robust, each one of these games $G_{k}$ has $k^{2}$ players.

The goal of this paper is to provide improvements by means of different experiments to the problem described above. In particular, we will classify all complete games (a basic class of games) of less than eight players according to they are: a weighted voting game or a game which is $(k-1)$-trade robust but not $k$-trade robust for all values of $k$. As a consequence it will we showed the existence of games with less than $k^{2}$ players which are $(k-1)$-trade robust but not $k$-trade robust. We want to point out that the classifications obtained in this paper by means of experiments are new in the mentioned fields.
\end{abstract}

\section{Introduction}

Simple games can be viewed as models of voting systems in which a single alternative, such as a bill or an amendment, is pitted against the status quo.

Definition 1. A simple game $G$ is a pair $(N, \mathcal{W})$ in which $N=\{1,2, \ldots, n\}$ and $\mathcal{W}$ is a collection of subsets of $N$ that satisfies: $N \in \mathcal{W}, \emptyset \notin \mathcal{W}$ and (monotonicity) $S \in \mathcal{W}$ and $S \subseteq T \subseteq N$ then $T \in \mathcal{W}$.

Any set of voters is called a coalition, and the set $N$ is called the grand coalition. Members of $N$ are called players or voters, and the subsets of $N$ that are in $\mathcal{W}$ are called winning coalitions. The intuition here is that a set $S$ is a winning

\footnotetext{
* This research was supported by the Spanish "Ministerio de Ciencia y Tecnología" programme TIC2002-00190 (AEDRI II), and Grant BFM 2003-01314 of the Science and Technology Spanish Ministry and the European Regional Development Fund.
} 
coalition iff the bill or amendment passes when the players in $S$ are precisely the ones who voted for it. A subset of $N$ that is not in $\mathcal{W}$ is called a losing coalition. A minimal winning coalition is a winning coalition all of whose proper subsets are losing. Because of monotonicity, any simple game is completely determined by its set of minimal winning coalitions. Before proceeding, we introduce some real-world examples of simple games (see Taylor [7] for more details on these examples).

Example 1. The European Economic Community (1958). In 1958, the Treaty of Rome established the existence of a binary voting system called the European Economic Community. The voters in this system were the following six countries: France, Germany, Italy, Belgium, the Netherlands and Luxembourg. France, Germany and Italy were given four votes each, while Belgium and the Netherlands were given two votes and Luxembourg one. Passage required a total quota of at least twelve of the seventeen votes. The European Economic Community was altered several times with the addition of new countries and a reallocation of votes. The current version was approved in the Nice European summit, held in December 2000.

Example 2. The United Nations Security Council. The voters in this system are the fifteen countries that make up the Security Council, five of which are called permanent members whereas the other ten are called non-permanent members. Passage requires a total of at least nine of the fifteen possible votes, subject to a veto due to a nay vote from any one of the five permanent members. This example ignores abstention. For a treatment of this example considering the possibility of abstention we refer the reader to [4].

Example 3. The United States Federal System. There are 537 voters in this system: 435 members of the House of Representatives, 100 members of the Senate, the vice president, and the president. The vice president plays the role of tiebreaker in the Senate, and the president has veto power that can be overridden by a two-thirds vote of both the House and the Senate. Thus, for a bill to pass it must be supported by either: 218 or more representatives and 51 or more senators (with or without the vice president) and the president; 218 or more representatives and 50 senators and the vice president and the president; or 290 or more representatives and 67 or more senators (with or without either the vice president or the president).

Example 4. The System to Amend the Canadian Constitution. Since 1982, an amendment to the Canadian Constitution can become law only if it is approved by at least seven of the ten Canadian provinces, subject to the proviso that the approving provinces have, among them, at least half of Canada's population. It was first studied in Kilgour [6]. A census (in percentages) for the the Canadian provinces was: Prince Edward Island (1\%), Newfoundland (3\%), New Brunswick (3\%), Nova Scotia (4\%), Manitoba (5\%), Saskatchewan (5\%), Alberta (7\%), British Columbia (9\%), Quebec (29\%) and Ontario (34\%).

For example observe that coalitions (from now on we make use of abridgements to denote the province) $S_{1}=\{P E I$, New, Man, Sas, Alb, BC, Que $\}$ and 
$S_{2}=\{N B, N S$, Man, Sas, Alb, BC, Ont $\}$ are minimal winning coalitions because they both have exactly 7 provinces and their total population surpasses the 50\%. Instead, coalitions $T_{1}=\{$ Man, Sas, Alb, BC, Que, Ont $\}$ and $T_{2}=$ $\{P E I, N e w, N B, N S$, Man, Sas, Alb, BC $\}$ are both losing because $T_{1}$ does not have 7 members and $T_{2}$ does not reach the $50 \%$ of the total Canada's population.

\section{Preliminaries}

\subsection{Weighted simple games}

Of fundamental importance to simple games are the subclasses of weighted simple games and complete simple games.

Definition 2. A simple game $G=(N, \mathcal{W})$ is said to be weighted if there exists a "weight function" $w: N \rightarrow \mathbb{R}$ and a real number "quota" $q \in \mathbb{R}$ such that a coalition $S$ is winning precisely when the sum of the weights of the players in $S$ meets or exceeds the quota.

The associated weight vector is $\left(w_{1}, \ldots, w_{n}\right)$. Any specific example of such a weight function $w: N \rightarrow \mathbb{R}$ and quota $q$ as in Definition 2 are said to realize $G$ as a weighted game. A particular realization of a weighted simple game is denoted as $\left[q ; w_{1}, \ldots, w_{n}\right]$.

Example 1 corresponds to $[12 ; 4,4,4,2,2,1]$ where 4 is the weight assigned to France, Germany and Italy, 2 the weight assigned to Belgium and the Netherlands and 1 the weight assigned to Luxembourg. A realization of Example 2 is $[39 ; 7,7,7,7,7,1,1,1,1,1,1,1,1,1,1]$ where 7 is the weight for a permanent member and 1 the weight for a non-permanent member.

Simple games and in particular weighted games and complete games, which we will introduce later on, have been studied in a variety of different mathematical contexts: Boolean or switching functions, threshold logic, hypergraphs, coherent structures, Sperner systems, and clutters. One of the most important problems for all those fields is determining whether a simple game can be realized as a weighted simple game. The only results giving necessary and sufficient conditions can be found under one of the next three topics: geometric approach based on separating hyperplanes; algebraic approach based on systems of linear inequalities; approach based on trading transforms. The geometric approach requires translating the question of weightedness into one of separability via a hyperplane of two convex subsets of $\mathbb{R}^{n}$. The key idea in the algebraic approach involves translating weightedness via vector sums into conditions equivalent to the solvability of systems of linear inequalities. The approach based on trades is the most natural and suggests several interpretations that will be tackled here from a computational viewpoint.

Definition 3. Suppose $G=(N, \mathcal{W})$ is a simple game. Then a trading transform (for $G$ ) is a coalition sequence $\mathcal{J}=\left\langle S_{1}, \ldots, S_{j}, T_{1}, \ldots, T_{j}\right\rangle$ (from $G$ ) of even length satisfying the following condition: $\left|\left\{i: p \in S_{i}\right\}\right|=\left|\left\{i: p \in T_{i}\right\}\right|$ for all $p \in N . S_{i}$ are called the pre-trade coalitions and the $T_{i}$ the post-trade coalitions, and we will say that $\left\langle S_{1}, \ldots, S_{j}\right\rangle$ has been converted by a trade to $\left\langle T_{1}, \ldots, T_{j}\right\rangle$. 
Definition 4. A $k$-trade for a simple game $G$ is a trading transform $\mathcal{J}=$ $\left\langle S_{1}, \ldots, S_{j}, T_{1}, \ldots, T_{j}\right\rangle$ in which $j \leq k$. The simple game $G$ is $k$-trade robust if there is no such $\mathcal{J}$ for which all the $S$ s are winning in $G$ and all the $T$ s are losing in $G$. If $G$ is $k$-trade robust for all $k$, then $G$ is said to be trade robust.

Loosely speaking, $G$ is $k$-trade robust if a sequence of $k$ or fewer (not necessarily distinct) winning coalitions can never be rendered losing by a trade.

Theorem 1. (Taylor and Zwicker, [8]). For a simple game $G=(N, \mathcal{W})$, the following are equivalent:

(i) $G$ is weighted. (ii) $G$ is trade robust. (iii) $G$ is $2^{2^{|N|}}$-trade robust.

Notice that a naive checking of (iii) is a finite (albeit lengthy) process, whereas a naive checking of weightedness directly is an infinite process. Moreover, Theorem 1 actually provides a fairly simple and uniform procedure for showing that certain games are not weighted: one produces a sequence of winning coalitions and indicates trades among these winning coalitions that convert all of them to losing coalitions.

In Example 4 we have seen that the trading transform $\mathcal{J}=\left\langle S_{1}, S_{2}, T_{1}, T_{2}\right\rangle$ converts the winning coalitions $S_{1}$ and $S_{2}$ to the losing ones $T_{1}$ and $T_{2}$. Therefore, by Theorem 1, the system is not weighted. Another complex voting system is the current European Economic Community. The countries are: Germany, United Kingdom, France, Italy, Spain, Poland, Romania, The Netherlands, Greece, Czech Republic, Belgium, Hungary, Portugal, Sweden, Bulgaria, Austria, Slovak Republic, Denmark, Finland, Ireland, Lithuania, Latvia, Slovenia, Estonia, Cyprus, Luxemburg and Malta. They are represented by the set $\{1,2, \ldots, 27\}$ where Germany $=1$, United Kingdom $=2$, and so on.

The first decision rule is the simple game given by $v_{1} \cap v_{2} \cap v_{3}$, where the three weighted voting games corresponding to votes, countries and population, are the following:

$$
\begin{aligned}
& v_{1}=[255 ; 29,29,29,29,27,27,14,13,12,12,12,12,12,10,10,10,7,7,7,7,7,4,4,4,4,4,3] \\
& v_{2}=[14 ; 1,1,1,1,1,1,1,1,1,1,1,1,1,1,1,1,1,1,1,1,1,1,1,1,1,1,1], \\
& v_{3}=[620 ; 170,123,122,120,82,80,47,33,22,21,21,21,21,18,17,17,11,11,11,8,8,5,4,3,2,1,1] .
\end{aligned}
$$

Freixas [2] proves that this system cannot be expressed as intersection of only one or two voting systems. To see that it fails to be 2 -trade robust, we may consider coalitions $S_{1}=T_{1} \backslash\{14,15,17\} \cup\{3\}$ and $S_{2}=T_{2} \backslash\{3\} \cup\{14,15,17\}$ where $T_{1}=[1,2] \cup[5,19] \cup\{26\}$ and $T_{2}=[1,13]$ (if $i \leq j$ we write $[i, j]=\{k \in$ $N: i \leq k \leq j\})$. The corresponding weights are:

\begin{tabular}{cc}
$v_{1} v_{2} v_{3}$ & $v_{1} v_{2} v_{3}$ \\
\hline$S_{1} 25616803$ & $T_{1} 25418727$ \\
$S_{2} 25515897$ & $T_{2} 25713883$
\end{tabular}

So after trades, the losing coalitions $T_{1}$ and $T_{2}$ in $v$ convert to the winning coalitions $S_{1}$ and $S_{2}$; consequently, game $v$ cannot be weighted because, after the trade, $S_{1}$ and $S_{2}$ cannot simultaneously gain weight.

The question of whether any bounded amount of trade robustness implies weightedness was settled by Taylor and Zwicker. 
Theorem 2. (Taylor and Zwicker, [9]) For each integer $k \geq 3$, there exists a simple game $G_{k}$ with $k^{2}$ players, that is $(k-1)$-trade robust, but not $k$-trade robust.

It will be of interest checking whether it exists a game with less than 9 players being 2 -trade but not 3-trade, or a game with less than 16 players being 2 and 3 trade robust but not 4 -robust. If the answer to these questions were affirmative then it would be of interest determining the minimum number of voters needed to reach games within these categories. In this paper we will make experiments in order to solve this problem for some values. We are also interested in studying other cases where the game shows a considerably symmetry among voters.

Unfortunately the number of simple games is too large to be tackled straightforwardly. We introduce another significant class of simple games that will help us to face our problem.

\subsection{Complete simple games}

Definition 5. Suppose $(N, \mathcal{W})$ is a simple game. Then $G$ is said to be swap robust if a one-for-one exchange between two winning coalitions can never render both losing.

Thus, swap robustness differs from trade robustness in two ways: the trades involve only two coalitions, and the exchanges are one for one. It is fairly easy to generate simple games that are not swap robust. A real-world example is the U.S. federal legislative system described in Example 3 where we may choose two coalitions formed respectively by: (a) 218 representatives, 51 senators and the president; (b) 290 representatives and 67 senators. Both coalitions are winning, but assume that simultaneously one senator belonging only to the first coalition moves to the second coalition, and, one representative only present in the second coalition moves to the first coalition. After these switches the coalitions have been converted respectively to: (a) 219 representatives, 50 senators and the president; (b) 289 representatives and 68 senators; and both coalitions become losing showing a of swap robustness and so a failure of 2-trade robustness. Note that this also means that the voting system is not weighted. Let us consider the following relations.

Definition 6. Let $(N, \mathcal{W})$ be a simple game, $i$ and $j$ be two voters. Players $i$ and $j$ are said to be equally desirable, denoted by $i \sim j$ if: for any coalition $S$ such that $i \notin S$ and $j \notin S, S \cup\{i\} \in \mathcal{W} \Leftrightarrow S \cup\{j\} \in \mathcal{W}$.

Definition 7. (Isbell, [5]) Let $(N, \mathcal{W})$ be a simple game, $i$ and $j$ be two voters. Player $i$ is said to be more desirable than $j$, denoted by $i \succ j$ if the following two conditions are fulfilled:

1. For every coalition $S$ such that $i \notin S$ and $j \notin S, S \cup\{j\} \in \mathcal{W} \Rightarrow S \cup\{i\} \in \mathcal{W}$.

2. There exists a coalition $T$ such that $i \notin T$ and $j \notin T, T \cup\{i\} \in \mathcal{W}$ and $T \cup\{j\} \notin \mathcal{W}$. 
The desirability relation denoted by $\succeq$ is defined in $N$ as follows: $i \succeq j$ if $i \succ j$ or $i \sim j$, we say that $i$ is at least as desirable as $j$ as coalitional partner. It is straightforward to check that $\sim$ is an equivalence relation, and that $\succeq$ is a partial ordering of the resulting equivalence classes.

Definition 8. A simple game $(N, \mathcal{W})$ is complete or linear if the desirability relation is a complete preordering.

In a complete simple game we may decompose $N$ in a collection of subsets, called classes, $N_{1}>N_{2}>\cdots>N_{t}$ forming a partition of $N$ and understanding that if $i \in N_{p}$ and $j \in N_{q}$ then: $p=q$ iff $i \sim j$ and $p<q$ if $i \succ j$. The following is a characterization of complete simple games.

Theorem 3. (Taylor and Zwicker, [10]) $G$ is a complete simple game iff $G$ is swap robust.

Because trade robustness implies swap robustness it may be concluded that if a simple game is weighted then it is complete. Carreras and Freixas [1] provide a classification theorem for complete simple games that allow to enumerate all these games up to isomorphism by listing the possible values of certain invariants. Previously to state it we need some preliminaries.

If $\bar{n}=\left(n_{1}, \ldots, n_{t}\right) \in \mathbb{N}^{t}$, we define $\Lambda(\bar{n})=\left\{\bar{m} \in(\mathbb{N} \cup\{0\})^{t}: \bar{m} \leq \bar{n}\right\}$ the set of all vectors $\bar{m}=\left(m_{1}, \ldots, m_{t}\right)$ whose components satisfy $0 \leq m_{k} \leq n_{k}$ for all $k=1, \ldots, t$ with the ordering $\delta$ given by the comparison of partial sums; that is,

$$
\bar{m} \delta \bar{p} \text { iff } \sum_{i=1}^{k} m_{i} \geq \sum_{i=1}^{k} p_{i} \text { for } k=1,2, \ldots, t .
$$

If $\bar{m} \delta \bar{p}$ we will say that $\bar{m} \delta$-dominates $\bar{p}$. If $\bar{m} \not \bar{p}$ and $\bar{p} \phi \bar{m}$ we will say that $\bar{m}$ and $\bar{p}$ are not $\delta$-comparable. From now on, we shall write $\Sigma_{k}(\bar{m})=\sum_{i=1}^{k} m_{i}$ for $k=1,2, \ldots, t$ and $\Sigma(\bar{m})=\left(\Sigma_{1}(\bar{m}), \ldots, \Sigma_{t}(\bar{m})\right)$ so that $\bar{m} \delta \bar{p}$ iff $\Sigma(\bar{m}) \geq \Sigma(\bar{p})$. It is not difficult to check that the couple $(\Lambda(\bar{n}), \delta)$ is a distributive lattice. Finally, two simple games $(N, \mathcal{W})$ and $\left(N^{\prime}, \mathcal{W}^{\prime}\right)$ are said to be isomorphic if there is a bijective map $f: N \rightarrow N^{\prime}$ such that $S \in \mathcal{W}$ iff $f(S) \in \mathcal{W}^{\prime} ; f$ is called an isomorphism of simple games.

To make understandable the following theorem we need to introduce the lexicographical ordering by partial sums. If $h<h^{\prime}$, then there exists some $l$ such that $\Sigma_{k}\left(\bar{m}_{h}\right)=\Sigma_{k}\left(\bar{m}_{h^{\prime}}\right)$ for $k<l$ and $\Sigma_{l}\left(\bar{m}_{h}\right)>\Sigma_{l}\left(\bar{m}_{h^{\prime}}\right)$.

Theorem 4. (Carreras and Freixas, [1])

Part A Let $G=(N, \mathcal{W})$ be a complete simple game with nonempty classes $N_{1}>N_{2}>\cdots>N_{t}$, let $\bar{n}$ be the vector defined by their cardinalities, and let $\mathcal{M}=\left(m_{i, j}\right)$, with $1 \leq i \leq r$ and $1 \leq j \leq t$, be the matrix satisfying the four conditions below:

(1) $m_{i, j} \in \mathbb{N} \cup\{0\}$ and $0 \leq m_{i, j} \leq n_{i}$ for all $i, j$ with $1 \leq i \leq r$ and $1 \leq j \leq t$;

(2) every pair of rows of $\mathcal{M}, \bar{m}_{h}$ and $\bar{m}_{h^{\prime}}$ are not $\delta$-comparable if $h \neq h^{\prime}$; 
(3) if $t=1$ then $m_{1,1}>0$; if $t>1$ then for every $k<t$ there exists some $h$ such that $m_{h, k}>0$ and $m_{h,(k+1)}<n_{k+1}$; and

(4) the rows of $\mathcal{M}$ are lexicographically ordered by partial sums.

Part B (Uniqueness) Two complete simple games $(N, \mathcal{W})$ and $\left(N^{\prime}, \mathcal{W}^{\prime}\right)$ are isomorphic iff $\bar{n}=\bar{n}^{\prime}$ and $\mathcal{M}=\mathcal{M}^{\prime}$.

Part C (Existence) Given a vector $\bar{n}$ and a matrix $\mathcal{M}$ satisfying the conditions of part $A$, there exists a complete simple game $(N, \mathcal{W})$ the characteristic invariants of which are $\bar{n}$ and $\mathcal{M}$.

We need now to describe how to get $(\bar{n}, \mathcal{M})$ from $(N, \mathcal{W})$ and reciprocally. As $(N, \mathcal{W})$ is a complete simple game either $i \succ j$, or $i \sim j$, or $j \succ i$ for all pair of voters. Voters being equally desirable are grouped in classes $N_{k}$, and the notation $N_{p}>N_{q}$ means that $i \succ j$ for each $i \in N_{p}$ and $j \in N_{q}$, Let $N_{1}>N_{2}>\cdots>N_{t}$. Components of vector $\bar{n}$ are defined by $n_{k}=\left|N_{k}\right|$. Rows of $\mathcal{M}$ are obtained in the following way, for each $S \in \mathcal{W}$ we consider the associated vector $\bar{s} \in \Lambda(\bar{n})$ with components $s_{k}=\left|S \cap N_{k}\right|, \bar{s}$ is a row of $\mathcal{M}$ if it is not dominated for any other vector associated to a winning coalition. Once the collection of non-dominated vectors corresponding to winning coalitions is determined we need to order them lexicographically. Reciprocally, given $\bar{n}$ and $\mathcal{M}$ let $n=\Sigma_{t}(\bar{n})=n_{1}+n_{2}+\cdots+n_{t}$ be the number of players, $N=\{1,2, \ldots, n\}$ be the set of players, and $N_{1}, N_{2}, \ldots, N_{t}$ be subsets of $N$ formed, respectively, by $n_{1}, n_{2}, \ldots, n_{t}$ elements (which may be chosen following the natural ordering). By Theorem 4(A), none of these subsets is empty. For each $S \subseteq N$ we consider $\bar{s}=\left(s_{1}, s_{2}, \ldots, s_{t}\right)$, where $s_{k}=\left|S \cap N_{k}\right|$ for $k=1,2, \ldots, t$. Then, the set of winning coalitions is $\mathcal{W}=\left\{S \subseteq N: \bar{s} \delta \bar{m}_{h}\right.$ for some row $\bar{m}_{h}$ of $\left.\mathcal{M}\right\}$.

Theorem 4 is a parametrization theorem, because it allows one to enumerate all complete games up to isomorphism by listing the possible values o certain invariants. Let us consider the examples previously introduced, we may easily deduce that the characteristic invariants of Example 1 are:

$$
\bar{n}=(3,2,1), \quad \mathcal{M}=\left(\begin{array}{lll}
3 & 0 & 0 \\
2 & 2 & 0
\end{array}\right)
$$

The characteristic invariants for Example 2 are: $\bar{n}=(5,10), \quad \mathcal{M}=(54)$. For Example 4 we obtain $\bar{n}=(2,8), \quad \mathcal{M}=(16)$. Oppositely, Example 3 is not complete so cannot be represented by means of the characteristic invariants. Although the voting system of the current European Economic Community is complete its representation using characteristic invariants is a bit complex.

\section{Experiments on the complex simple games}

\subsection{A new theoretical approach to performance experiments}

In this subsection we reformulate the developed theory in the preceding section in order to deal with it from a more efficient computational viewpoint. For lack of space, we just give here the main ideas of some new theoretical results. 
In general, to describe a simple game it is enough giving the list of minimal winning coalitions. If moreover, the game is complete and the ordering induced by the desirability relation among components is known, then we can use a subset of minimal winning coalitions to entirely describe the game. Indeed, a coalition $S$ such that its associated vector $\bar{s}$ is a row of $\mathcal{M}$ is called a $\delta$-minimal winning coalition. Notice that each $\delta$-minimal winning coalition is a minimal winning coalition but the reciprocal is not true. For instance, in Example 4 a coalition formed by Ontario, Quebec and 5 more provinces is minimal winning but not $\delta$-minimal winning since its associated vector $(2,5)$ is not a row of matrix $\mathcal{M}$.

For the purpose of studying when a game is weighted in terms of trade robustness we may confine to study only complete simple games, because the remaining simple games are not swap robust which is the simplest case of not being 2-trade robust. Within the framework of complete simple games we can take advantage of using the equivalent representation $(\bar{n}, \mathcal{M})$, which allows using models of coalitions instead of coalitions and considering only models which are rows of matrix $\mathcal{M}$. These latter properties become essential in this section devoted to algorithms and experiments. The basic idea is that it simplifies the description of the algorithms as well as it meaningfully improves the performance of the experiments.

Definition 9. (cf. Definition 3) Suppose $G=(N, \mathcal{W})$ is a simple game. Then a $\delta$-trading transform (for $G$ ) is a coalition sequence $\mathcal{J}=\left\langle S_{1}, \ldots, S_{j}, T_{1}, \ldots, T_{j}\right\rangle$ (from $G$ ) of even length satisfying condition $\left|\left\{i: p \in S_{i}\right\}\right|=\left|\left\{i: p \in T_{i}\right\}\right|$ for all $p \in N$, where $S_{1}, \ldots, S_{j}$ are $\delta$-minimal winning coalitions.

Definition 10. (cf. Definition 4) $A k$ - $\delta$-trade for a simple game $G$ is a $\delta$-trading transform $\mathcal{J}=\left\langle S_{1}, \ldots, S_{j}, T_{1}, \ldots, T_{j}\right\rangle$ in which $j \leq k$. The simple game $G$ is $k$ - $\delta$-trade robust if there is no such $\mathcal{J}$ for which all the $S$ s are $\delta$-minimal winning coalitions in $G$ and all the $T$ s are losing in $G$. If $G$ is $k$ - $\delta$-trade robust for all $k$, then $G$ is said to be $\delta$-trade robust.

Proposition 1. Let $G=(N, \mathcal{W})$ be a complete game. Then, $G$ is $k$-trade robust iff $G$ is $k$ - $\delta$-trade robust.

In the following, we provide a trading version applied to indices of columns of $\mathcal{M}$ and vectors instead of players and coalitions.

Definition 11. Let $G=(N, \mathcal{W})$ be a complete simple game with characteristic invariants $(\bar{n}, \mathcal{M})$. A vectorial trading transform for $G$ is a vectorial sequence $\mathcal{J}^{\prime}=\left\langle\bar{x}_{1}, \ldots, \bar{x}_{j}, \bar{y}_{1}, \ldots, \bar{y}_{j}\right\rangle$ of even length satisfying the following conditions:

$$
\sum_{j=1}^{r} x_{i, j}=\sum_{j=1}^{r} y_{i, j} \quad \forall i \in[1, t]
$$

where $\bar{y}_{1}, \ldots, \bar{y}_{j}$ belong to $\Lambda(\bar{n})$ and, moreover, $\bar{x}_{1}, \ldots, \bar{x}_{j}$ are rows of $\mathcal{M}$ with repetitions allowed. 
Definition 12. Let $G=(N, \mathcal{W})$ be a complete simple game with characteristic invariants $(\bar{n}, \mathcal{M})$. Then, $G$ is $k$-invariant-trade robust ( $k-I-T-R$, for short) if there is no a vectorial trading transform $\mathcal{J}^{\prime}=\left\langle\bar{x}_{1}, \ldots, \bar{x}_{j}, \bar{y}_{1}, \ldots, \bar{y}_{j}\right\rangle$ such that each $\bar{x}_{i}$ is a row of $\mathcal{M}$ and each $\bar{y}_{k} \in \Lambda(\bar{n})$ for $1 \leq k \leq j$ satisfies $\bar{y}_{k} \phi \bar{m}_{i}$ for every row $\bar{m}_{i}$ of $\mathcal{M}$. If $G$ is $k-I-T-R$ for all positive integer $k$, then $(N, \mathcal{W})$ is invariant-trade robust ( $I-T-R$, for short).

The following proposition states that Definitions 11 and 12 merely correspond to Definitions 9 and 10 if we consider the context $(\bar{n}, \mathcal{M})$ instead of $(N, \mathcal{W})$. We omit the proof because applying Proposition 1 it follows that $k$-trade robust is equivalent to $k$ - $\delta$-trade robust and word-by-word this is equivalent to $k$-I-T-R.

Proposition 2. Let $G=(N, \mathcal{W})$ be a simple game with characteristic invariants $(\bar{n}, \mathcal{M})$. Then, $(N, \mathcal{W})$ is $k$-trade robust iff $(\bar{n}, \mathcal{M})$ is $k-I-T-R$.

Theorem 1 by Taylor and Zwicker for simple games can be adapted to complete simple games.

Theorem 5. Let $G$ be a complete simple game $(N, \mathcal{W})$ with characteristic invariants $(\bar{n}, \mathcal{M})$ and $t$ being the number of columns if $\mathcal{M}$. Then,

(i) $G$ is weighted. (ii) $G$ is invariant-trade robust. (iii) $G$ is $2^{2^{t}}-I-T-R$.

\subsection{A full classification for complete simple games with less than eight voters}

All experiments we have made are based on Theorem 5. It gives a new viewpoint to determine if a complete simple game is trade robust. Our programs ${ }^{1}$ have been written for $\mathrm{C}++$ and run under Linux in Pentium 4 at $1.7 \mathrm{GHz}$ with $512 \mathrm{Mb}$ of RAM. To set an example, we just sketch here one of the implemented algorithms. So, Algorithm 1 sketches the used recursive function (based on backtracking method) to determine if a given simple game with characteristic invariants $(\bar{n}, \mathcal{M})$ is $k$-I-T-R or not. The parameters of function DoingForkTrade mean the following: $\mathcal{M}$ and $\bar{n}$ are the given matrix and vector, $r$ and $t$ are the number of computed rows and columns for the current matrix $\mathcal{Y}=\left(y_{i, j}\right),{ }^{2}$ and finally, the auxiliary parameter aux is used to improve the algorithm. This function calls three additional functions:

- Function CanonicalMatrix $(\mathcal{Y})$ returns true if the rows of $\mathcal{Y}$ are in lexicographic order (by rows and by columns), false otherwise.

- Function AnyRowOfYDominates $M(\mathcal{Y}, \mathcal{M})$ returns true if any row of $\mathcal{Y}$ dominates at least one row of $\mathcal{M}$, false otherwise.

- Function PartialSums $\left(\mathcal{M}, i_{r}, e_{r}, i_{t}, e_{t}\right)$ adds up the integers of $\mathcal{M}$ from row $i_{r}$ to row $e_{r}$ and from column $i_{t}$ to column $e_{t}$.

In general, given $(\bar{n}, \mathcal{M})$ and a positive integer $k$, calling the recursive function DoingForkTrade $(\mathcal{M}, \mathcal{Y}, \bar{n}, 1,1, \operatorname{PartialSums}(\mathcal{M}, 1, k, 1,1)$ ), it returns true (Line 20 ) if it is $k$-I-T-R, and false (Line 7 ) otherwise.

\footnotetext{
1 They are available on request from the authors.

${ }^{2}$ We denote by $\mathcal{Y}$ any of the matrices with rows $\bar{y}_{1}, \ldots, \bar{y}_{j}$ that are solution of equation in Definition 11, showing a failure of $j$-trade robust.
} 


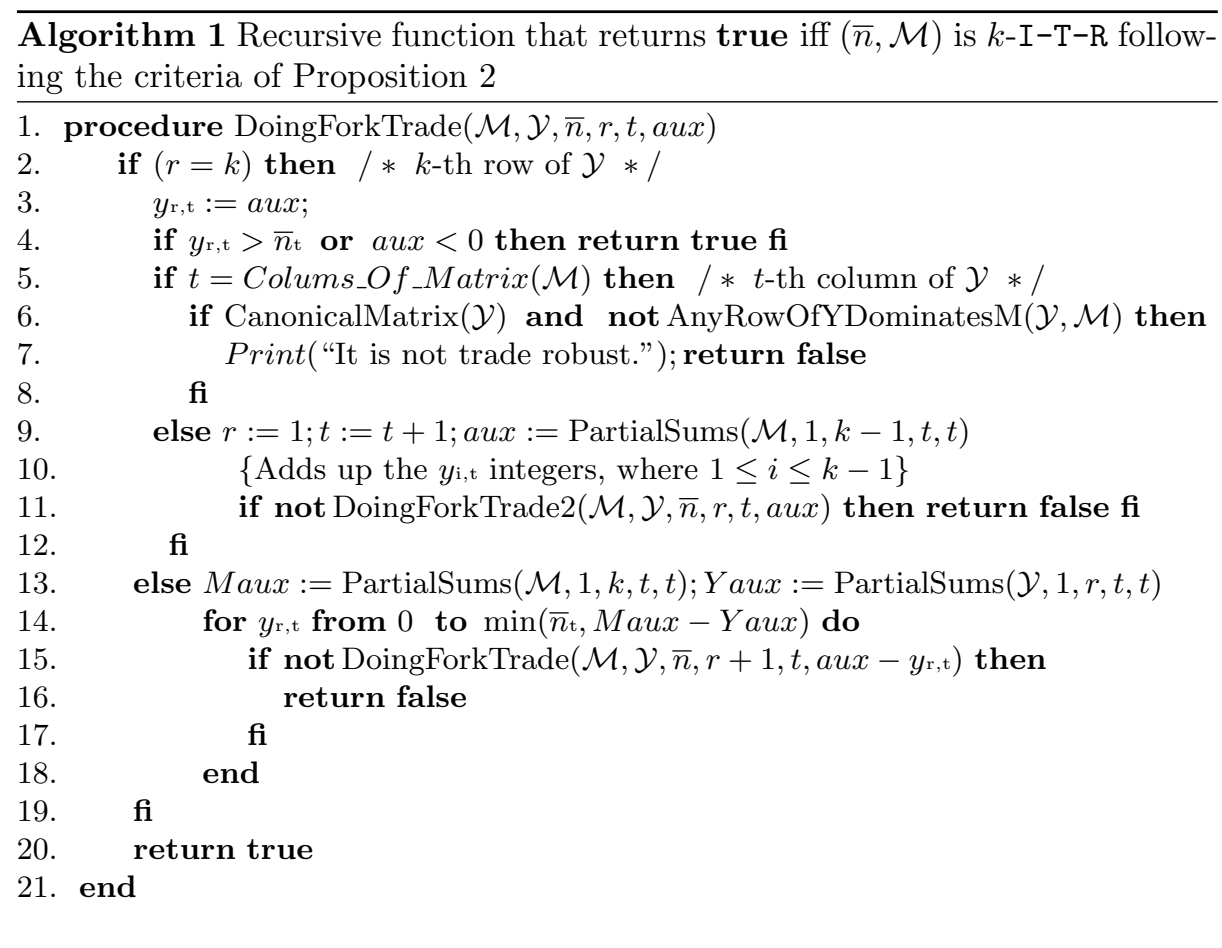

Unfortunately, the number of matrices associated to a fixed number $n$ of voters is huge for $n>8$. However, the experiments are successful for a small fixed number of columns (two or three) and for small numbers of voters $(n<9)$.

Table 1 provides a detailed classification of all complete simple games: the number of complete games (briefly $C G$ ), the number of weighted games (briefly $W G)$, and the number of non $k$-invariant-trade robust but $(k-1)$-invariant-trade robust games (non $k$-I-T-R, for short). Finally, the number of complete games being non weighted is gathered in non invariant-trade robust games (I-T-R, for short).

\begin{tabular}{crrrrrrr}
$n$ & 1 & 2 & 3 & 4 & 5 & 6 & 7 \\
\hline$C G$ & 1 & 3 & 8 & 25 & 117 & 1171 & 44313 \\
$W G$ & 1 & 3 & 8 & 25 & 117 & 1111 & 29375 \\
non $I-T-R$ & 0 & 0 & 0 & 0 & 0 & 60 & 14938 \\
\hline non $2-I-T-R$ & 0 & 0 & 0 & 0 & 0 & 57 & 13915 \\
non $3-I-T-R$ & 0 & 0 & 0 & 0 & 0 & 3 & 1011 \\
non $4-I-T-R$ & 0 & 0 & 0 & 0 & 0 & 0 & 14 \\
\hline
\end{tabular}

Table 1. Full classification of simple games for $n<8$

In particular, $n=6$ is the minimum number of voters required to achieve simple games which are $2-\mathrm{I}-\mathrm{T}-\mathrm{R}$ but not $3-\mathrm{I}-\mathrm{T}-\mathrm{R} ; n=7$ is the minimum number 
of voters required to achieve simple games which are 3-I-T-R but not 4-I-T-R. Tables 4 and 5 in Appendix enumerate all these extreme cases giving vector $\bar{n}$, matrix $\mathcal{M}$ and a matrix $\mathcal{Y}$ which fulfills equation in Definition 11 and shows a failure to be $k$-I-T-R: $k=3$ for $n=6$, and $k=4$ for $n=7$.

\subsection{A detailed analysis for simple games with two and three columns}

The huge number of simple games for more than 8 voters does not allow to consider all simple games in a reasonable time. However, fixing a small number of columns (two or three) we can do an exhaustive experimental study even for 10 voters. In terms of simple games (voting systems) few columns mean the existence of many equally desirable voters which is highly frequent.

For two columns, we have checked that all simple games with $n \leq 10$ are either I-T-R or non 2-I-T-R (see Table 2). For three columns we also have found the full classification (see Table 3). We do not show particular examples because of the lack of space.

\begin{tabular}{crrrrrrrrrr}
$n$ & 1 & 2 & 3 & 4 & 5 & 6 & 7 & 8 & 9 & 10 \\
\hline$C G$ & 0 & 1 & 5 & 15 & 36 & 76 & 148 & 273 & 485 & 839 \\
\hline non 2-I-T-R & 0 & 0 & 0 & 0 & 0 & 2 & 10 & 34 & 94 & 229 \\
non 3-I-T-R & 0 & 0 & 0 & 0 & 0 & 0 & 0 & 0 & 0 & 0 \\
\hline
\end{tabular}

Table 2. Number of non $k$-I-T-R simple games with just two columns

\begin{tabular}{crrrrrrrrrr}
$n$ & 1 & 2 & 3 & 4 & 5 & 6 & 7 & 8 & 9 & 10 \\
\hline$C G$ & 0 & 0 & 0 & 6 & 50 & 262 & 1114 & 4278 & 15769 & 58147 \\
\hline non 2-I-T-R & 0 & 0 & 0 & 0 & 0 & 6 & 130 & 1116 & 6858 & 35431 \\
non 3-I-T-R & 0 & 0 & 0 & 0 & 0 & 0 & 6 & 39 & 160 & 506 \\
non 4-I-T-R & 0 & 0 & 0 & 0 & 0 & 0 & 2 & 11 & 39 & 115 \\
non 5-I-T-R & 0 & 0 & 0 & 0 & 0 & 0 & 0 & 0 & 2 & - \\
\hline
\end{tabular}

Table 3. Number of non $k$-I-T-R simple games with just three columns

\section{Conclusion and Future Work}

In this paper we have made experiments that allow:

(i) To classify all complete simple games, $C G$, for $n<8$ according to they are: $W G$, non 2-I-T-R, non 3-I-T-R and non 4-I-T-R.

(ii) To check if a particular complete simple game with $n$ voters is $k$-I-T-R for each positive integer $k$.

(iii) To study important subclasses, those with either one, two or three columns, whenever $n<11$.

The given results in $(i)$ for $n=6,7$ are new as well as the results obtained in (iii) for $n>5$. 
Our experiments suggest three important theoretical conjectures which we are developing.

Conjecture 1. Any simple game with just two types of voters (two classes) is either I-T-R or non 2-I-T-R.

Conjecture 2. Three columns are enough to find games which are $(k-1)-\mathrm{I}-\mathrm{T}-\mathrm{R}$ but non $k$-I-T-R for any positive integer $k$.

Conjecture 3. It is possible to find a game which is $(k-1)-\mathrm{I}-\mathrm{T}-\mathrm{R}$ but non $k$-I-T-R for any positive integer $k$, where the number of voters is $\mathrm{O}(k)$ instead of $k^{2}$ (cf. Theorem 2).

It is also interesting to study the required CPU time depending on the number of voters, the number of columns. Even, to do an exhaustive analysis depending on the number of rows (games with a single row are called complete simple games with minimum and have been studied in [3]).

Another future work is, for a fixed number of voters $n$, to generate a random game $(\bar{n}, \mathcal{M})$ and study trade robustness.

Finding appropriate weights for voters and a quota (a realization), for the class of $W G$.

\section{References}

1. F. Carreras and J. Freixas. Complete simple games. Mathematical Social Sciences, 32:139-155, 1996.

2. J. Freixas. The dimension for the European Union Council under the Nice rules. European Journal of Operational Research, 156(2):415-419, 2004.

3. J. Freixas and M.A. Puente. Complete games with minimum. Annals of Operations Research, 84:97-109, 1998.

4. J. Freixas and W.S. Zwicker. Weighted voting, abstention, and multiple levels of approval. Social Choice and Welfare, 21:399-431, 2003.

5. J.R. Isbell. A class of simple games. Duke Mathematics Journal, 25:423-439, 1958.

6. D.M. Kilgour. A formal analysis of the amending formula of Canada's Constitution. Act. Canadian Journal of Political Science, 16:771-777, 1983.

7. A.D. Taylor. Mathematics and Politics. Springer Verlag, New York, USA, 1995.

8. A.D. Taylor and W.S. Zwicker. A characterization of weighted voting. Proceedings of the American mathematical society, 115:1089-1094, 1992.

9. A.D. Taylor and W.S. Zwicker. Simple games and magic squares. Journal of combinatorial theory, ser. A, 71:67-88, 1995.

10. A.D. Taylor and W.S. Zwicker. Simple games: desirability relations, trading, and pseudoweightings. Princeton University Press, New Jersey, USA, 1999. 


\section{Appendix}

This appendix shows the specific examples which are $(k-1)-\mathrm{I}-\mathrm{T}-\mathrm{R}$ but non $k$-I-T-R for the highest value of $k$ and for $n=6,7$.

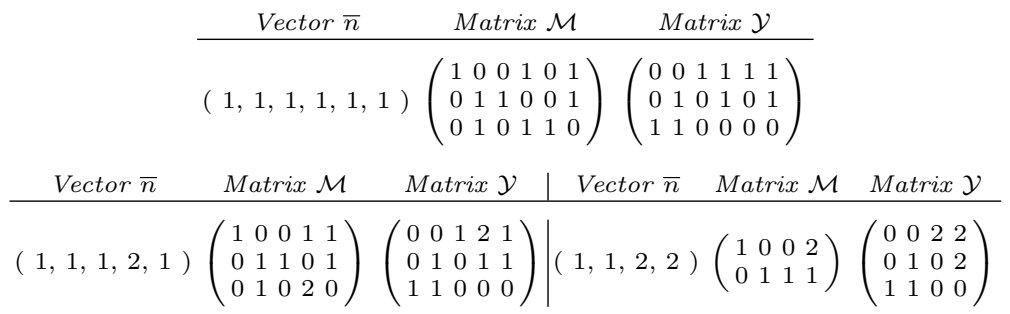

Table 4. Non 3-I-T-R simple games for $n=6$

\begin{tabular}{|c|c|c|c|c|c|c|c|c|}
\hline Vector $\bar{n}$ & Matrix $\mathcal{M}$ & Matrix $\mathcal{Y}$ & Matrix $\mathcal{M}$ & Matrix $\mathcal{Y}$ & Matrix $\mathcal{M}$ & Matrix $\mathcal{Y}$ & Matrix $\mathcal{M}$ & Matrix $\mathcal{Y}$ \\
\hline$(1,1,1,1,1,1,1)$ & $\left(\begin{array}{l}1010001 \\
1001010 \\
0101011 \\
0011101\end{array}\right)$ & $\left(\begin{array}{l}0011011 \\
0111000 \\
1000111 \\
1001001\end{array}\right)$ & & & & & & \\
\hline$(1,1,1,1,1,2)$ & $\left(\begin{array}{l}110002 \\
101011 \\
011102\end{array}\right)$ & $\left(\begin{array}{l}011012 \\
100112 \\
101002 \\
111000\end{array}\right)$ & & & & & & \\
\hline$(1,1,1,2,1,1)$ & $\left(\begin{array}{l}100010 \\
010011 \\
001101\end{array}\right)$ & $\left(\begin{array}{l}000211 \\
001011 \\
011000\end{array}\right)$ & & & & & & \\
\hline$(1,1,1,2,2)$ & $\left(\begin{array}{l}10002 \\
01102 \\
01021\end{array}\right)$ & $\left(\begin{array}{l}00122 \\
01012 \\
01012 \\
11000\end{array}\right)$ & & & & & & \\
\hline$(1,1,2,1,2)$ & $\left(\begin{array}{l}10011 \\
01102\end{array}\right)$ & $\left(\begin{array}{l}00212 \\
01012 \\
10002 \\
11000\end{array}\right)$ & $\left(\begin{array}{l}10002 \\
01011 \\
00202\end{array}\right)$ & $\left(\begin{array}{l}00112 \\
00112 \\
01002 \\
11000\end{array}\right)$ & $\left(\begin{array}{l}11010 \\
10012 \\
01102\end{array}\right)$ & $\left(\begin{array}{l}00212 \\
01012 \\
11001 \\
11001\end{array}\right)$ & $\left(\begin{array}{l}11010 \\
10200 \\
01102\end{array}\right)$ & $\left(\begin{array}{l}00212 \\
01200 \\
11001 \\
11001\end{array}\right)$ \\
\hline$(1,1,2,2,1)$ & $\left(\begin{array}{l}10021 \\
01101 \\
00220\end{array}\right)$ & $\left(\begin{array}{l}00211 \\
00211 \\
01021 \\
11000\end{array}\right)$ & $\left(\begin{array}{l}10101 \\
10020 \\
01021\end{array}\right)$ & $\left(\begin{array}{l}00221 \\
10011 \\
10011 \\
11000\end{array}\right)$ & & & & \\
\hline$(1,2,2,2)$ & $\left(\begin{array}{l}1010 \\
0102\end{array}\right)$ & $\left(\begin{array}{l}0200 \\
1001 \\
1001\end{array}\right)$ & $\left(\begin{array}{l}1102 \\
0221\end{array}\right)$ & $\left(\begin{array}{l}0212 \\
1022 \\
1200\end{array}\right)$ & & & & \\
\hline$(2,2,3)$ & $\left(\begin{array}{l}210 \\
103\end{array}\right)$ & $\left(\begin{array}{l}023 \\
201 \\
201 \\
201\end{array}\right)$ & & & & & & \\
\hline$(2,3,2)$ & $\left(\begin{array}{l}102 \\
031\end{array}\right)$ & $\left(\begin{array}{l}022 \\
022 \\
022 \\
200\end{array}\right)$ & & & & & & \\
\hline
\end{tabular}

Table 5. Non 4-I-T-R simple games for $n=7$ 